\title{
Die Frühgeborenenosteopenie - ein aktuelles Problem?
}

\section{Osteopenia of Prematurity - a Current Problem?}

\section{Gortner}

Bibliografie

DOI http://dx.doi.org/ 10.1055/s-0032-1306351 Klin Padiatr 2012; 224: 51-52 (c) Georg Thieme Verlag KG Stuttgart . New York ISSN 0300-8630

Korrespondenzadresse Prof. Dr. Ludwig Gortner Klinik für Allgemeine Pädiatrie und Neonatologie, Kliniken für Kinder- und Jugendmedizin Universitätsklinikum des Saarlandes

Gebäude 9

66421 Homburg/Saar

ludwig.gortner@uks.eu
Die Frühgeborenenosteopenie (Knochenmineralmangel des Frühgeborenen, in der angloamerikanischen Literatur osteopenia of prematurity) wurde bereits zum Ende der 40er-Jahre des vergangenen Jahrhunderts beschrieben und zog sich durch die medizinische Literatur als Erkrankung Frühgeborener, welche primär durch einen Mangel an Vitamin D erklärt wurde [9]. Diese Form der Frühgeborenenosteopenie, welche durchweg bei Frühgeborenen eines Gestationsalters von mehr als 32 Wochen beschrieben worden war, wurde abgelöst von einer Form, die im Wesentlichen sehr unreife Frühgeborene, d.h. Kinder einer Gestationsaltersgruppe von weniger als 32 Wochen, betraf [3]. Hier waren typische klinische und radiologische Symptome die Rippenfrakturen Betroffener, welche erstmals in den späten 70er-Jahren zum Teil mit anderen Begleiterkrankungen beschrieben wurden [5]. Danach kamen zahlreiche, systematische Studien in den 80erJahren zur Publikation, die einen Zusammenhang zwischen inadäquater Kalzium- und Phosphatzufuhr und der Entwicklung einer Frühgeborenenosteopenie mit hochgradiger Frakturneigung belegten [2]. Fälschlicherweise wurden diese Frakturen in einem Teil der Fälle einem Fehlverhalten des ärztlichen, des Pflege- bzw. des weiteren medizinischen Personals kausal zugeordnet und mit strafrechtlicher Verfolgung belegt [16].

Die Ursachen der Osteopenie bei sehr unreifen Frühgeborenen wurden von mehreren Arbeitsgruppen überzeugend dargelegt und lassen sich hinsichtlich ihrer Pathogenese dahingehend zusammenfassen, dass im Wesentlichen eine inadäquate geringe Kalziumzufuhr bei Formelernährung und eine inadäquate geringe Phosphatzufuhr bei Muttermilchernährung sehr unreifer Frühgeborener die wesentliche Ursache darstellen [10]. Daneben sind tubuläre Fehlfunktionen mit Phosphatverlust, bedingt durch Hypoxie oder toxische Einwirkungen auf die Niere, beschrieben [8]. Nach gründlichen Untersuchungen der Pathogenese der Frühgeborenenosteopenie bei o.g. Gruppe von Patienten wurde in zahlreichen kleineren Studien die Therapie dahingehend angegangen, durch eine entsprechende Supplementierung mit Kalzium bzw. Phosphat eine adäquate Mineralisation, gemessen z. B. mit der dualen Photonenabsorptionsdensitometrie, zu erreichen. Auch hier wurden zahlreiche kleinere Studien in den 80er- und 90er-Jahren publiziert $[4,7]$. Es wurden jedoch keine großen multizentrischen randomisierten Doppelblindstudien durchgeführt, wie dies z. B. für die Surfactanttherapie, die NO-Behandlung und andere relevante neonatologische Interventionen der Fall war. Die hieraus resultierende Heterogenität der Therapieempfehlungen bzw. des Monitorings der Therapie bedingen, dass bis in unsere Dekade trotz der schon sehr langen Erkenntnisse zu pathophysiologischen Zusammenhängen keine einheitlichen Therapieleitlinien hierzu publiziert wurden.

Dies mag einer der Gründe sein, weshalb die unterschiedlichen Empfehlungen hinsichtlich der Kalzium- und Phosphataufnahme für sehr unreife Frühgeborene gegeben werden:

Die Europäische Gesellschaft für Pädiatrische Gastroenterologie, Hepatologie und Ernährung (ESPEGHAN) legt hierbei wesentlich tiefere Kalziumsupplementierungs-Empfehlungen vor, als dies von Gruppen in klinisch kontrollierten Studien [1] eingesetzt wurde. Seitens der American Academy of Pediatrics liegt zu der Thematik keine Behandlungsempfehlung vor, lediglich die Vitamin-D-Therapie beim Frühgeborenen wird im letzten aktuellen Statement zur Rachitisprävention und dem Vitamin-D-Mangel gestreift [17]. Es kann daher erwartet werden, dass obschon die Problematik der schweren, mit Knochenbrüchen einhergehenden Osteopenie selten geworden ist, eine einheitliche Interpretation alter Studiendaten oder gänzlich neue Daten nicht zu erwarten sind. Auch auf diesem Hintergrund werden in einer Übersichtsarbeit sowie einer Beobachtungsstudie die individuell gesteuerte Kalzium- und Phosphattherapie zur Prävention der Frühgeborenenosteopenie in dem vorliegenden Band ausführlich aufgegriffen $[13,14]$.

Aufgrund der unterschiedlichen resorptiven Verhältnisse der behandelten Frühgeborenen für Kalzium/Phosphat in Abhängigkeit von entweder Muttermilch oder Formelernährung, der Interferenz mit mütterlichen Therapien, wie z.B. einer lang dauernden Magnesiumtokolyse [18], mit dem Resultat einer verminderten Knochenakquisition bei der Geburt bleibt festzustellen, dass neben den bekannten Variablen weitere Einflussgrößen auf den Kalzium- und Phosphatstoffwechsel bei rasch wachsenden sehr unreifen Frühgeborenen zu diskutieren sind. Mithin sind, basierend auf obigen Überlegungen, die individuell dosierte, mittels der Urinuntersuchung einfach im klinischen Alltag zu handhabende Kontrolle der Kalzium- und Phosphatsupplementierung ein gangbarer Weg, beim Fehlen großer kontrollierter Studien die Prävention der Frühgeborenenosteopenie umzusetzen. Obschon die langfristigen Konsequenzen einer Frühgeborenenosteopenie bislang nur in wenigen Studien adressiert wurden [12], erscheint dies der wirkliche Goldstandard, um die Sicherheit und Effizienz 
der gezielten Kalzium- und Phosphatsupplementierung, basierend auf den vorgeschlagenen Regeln, weiter und nachhaltig zu etablieren. Studien hierzu sollten die zuvor geschilderte komplexe Problematik mit Einflussvariablen unterschiedlicher prä- und postnataler Komponenten analog anderen Nachsorgestudien berücksichtigen, um valide Studienergebnisse zu ermöglichen [6]. Langfristige Konsequenzen der Therapie auf die Knochendichte können analog der entwicklungsneurologischen Studien $[11,15]$ als deren Teil zumindest in ausgewiesenen Zentren angegangen werden.

\section{Literatur}

1 Agostoni C, Buonocore G, Carnielli VP et al. Enteral nutrient supply for preterm infants: commentary from the European Society of Paediatric Gastroenterology, Hepatology and Nutrition Committee on Nutrition. J Pediatr Gastroenterol Nutr 2010; 50: 85-91

2 Bosley AR, Verrier-Jones ER, Campbell MJ et al. Aetiological factors in rickets of prematurity. Arch Dis Child 1980; 55: 683-686

3 Davies DP, Hughes CA, Moore JR. Rickets in preterm infants. Arch Dis Child 1978; 5: 88-90

4 De CM, Pieltain C, Rigo J. Body composition in preterm infants fed standard term or enriched formula after hospital discharge. Eur J Nutr 2002; 41: 177-182

5 Glasgow JF, Thomas PS. The osteodystrophy of prolonged obstructive liver disease in childhood. Acta Paediatr Scand 1976; 65: 57-64

6 Gortner L. Comment on growth of term small-for-gestational-age and appropriate-for-gestational-age neonates during the first month of life. Klin Padiatr 2011; 223: 57-58

7 Horsman A, Ryan SW, Congdon PJ et al. Bone mineral accretion rate and calcium intake in preterm infants. Arch Dis Child 1989; 64: 910-918
8 James JR, Congdon PJ, Truscott J et al. Osteopenia of prematurity. Arch Dis Child 1986; 61: 871-876

9 Klackenberg $G$. Three years' prophylaxis against rickets in prematures, with D2 massive dose. Acta Paediatr 1945; 32: 508-519

10 Kovar IZ, Mayne PD, Robbe I. Hypophosphataemic rickets in the preterm infant; hypocalcaemia after calcium and phosphorus supplementation. Arch Dis Child 1983; 58: 629-631

11 Lacher M, Froehlich S, von Schweinitz $D$ et al. Early and long term outcome in children with esophageal atresia treated over the last 22 years. Klin Padiatr 2010; 222: 296-301

12 McDevitt H, Tomlinson C, White MP et al. Changes in quantitative ultrasound in infants born at less than 32 weeks' gestation over the first 2 years of life: influence of clinical and biochemical changes. Calcif Tissue Int 2007; 81: 263-269

13 Maas C, Pohlandt F, Mihatsch WA et al. Prevention of bone mineral deficiency in premature infants: review of the literature with focus on monitoring of urinary calcium and phosphate. Klin Padiatr 2012; 224: $80-87$

14 Mihatsch WA, Trotter A, Pohlandt F. Calcium and phosphor intake in preterm infants: sensitivity and specifity of 6-hour urine samples to detect deficiency. Klin Padiatr 2012; 224: 61-65

15 Moll M, Schoning M, Golz R et al. 2-year follow-up examinations (Bayley II) in infants born at $<32$ weeks in a German perinatal center. Klin Padiatr 2011; 223: 251-254

16 Radkowski MA. The battered child syndrome: pitfalls in radiological diagnosis. Pediatr Ann 1983; 12: 894-903

17 Wagner CL, Greer FR. Prevention of rickets and vitamin D deficiency in infants, children, and adolescents. Pediatrics 2008; 122: 1142-1152

18 Yokoyama K, Takahashi N, Yada Y et al. Prolonged maternal magnesium administration and bone metabolism in neonates. Early Hum Dev 2010; 86: 187-191 